\title{
The relationship between frequency and severity of vaso-occlusive crises and health-related quality of life and work productivity in adults with sickle cell disease
}

\author{
Avery A. Rizio ${ }^{1} \cdot$ Menaka Bhor $^{2} \cdot$ Xiaochen Lin ${ }^{1} \cdot$ Kristen L. McCausland ${ }^{1} \cdot$ Michelle K. White $^{1} \cdot$ Jincy Paulose $^{2}$. \\ Savita Nandal ${ }^{2} \cdot$ Rashid I. Halloway ${ }^{3}$ Lanetta Bronté-Hall ${ }^{4}$
}

Accepted: 31 December 2019 / Published online: 13 January 2020

(c) The Author(s) 2020

\begin{abstract}
Purpose Patients with sickle cell disease (SCD) may experience sickle cell-related pain crises, also referred to as vasoocclusive crises (VOCs), which are a substantial cause of morbidity and mortality. The study explored how VOC frequency and severity impacts health-related quality of life (HRQoL) and work productivity.

Methods Three hundred and three adults with SCD who completed an online survey were included in the analysis. Patients answered questions regarding their experience with SCD and VOCs, and completed the Adult Sickle Cell Quality of Life Measurement Information System (ASCQ-Me) and the Workplace Productivity and Activity Impairment: Specific Health Problem (WPAI:SHP). Differences in ASCQ-Me and WPAI:SHP domains were assessed according to VOC frequency and severity.

Results Nearly half of the patient sample (47.2\%) experienced $\geq 4$ VOCs in the past 12 months. The most commonly reported barriers to receiving care for SCD included discrimination by or trouble trusting healthcare professionals (39.6\%, 33.3\%, respectively), limited access to treatment centers (38.9\%), and difficulty affording services (29.4\%). Patients with more frequent VOCs reported greater impacts on emotion, social functioning, stiffness, sleep and pain, and greater absenteeism, overall productivity loss, and activity impairment than patients with less frequent VOCs $(P<0.05)$. Significant impacts on HRQoL and work productivity were also observed when stratifying by VOC severity $(P<0.05$ for all ASCQ-Me and WPAI domains, except for presenteeism).

Conclusions Results from the survey indicated that patients with SCD who had more frequent or severe VOCs experienced deficits in multiple domains of HRQoL and work productivity. Future research should examine the longitudinal relationship between these outcomes.
\end{abstract}

Keywords Sickle cell disease $\cdot$ Vaso-occlusive crises $\cdot$ Health-related quality of life $\cdot$ Work productivity

Avery A. Rizio

arizio@qualitymetric.com

1 Patient Insights, Optum, 1301 Atwood Ave, Suite 311N, Johnston, RI, USA

2 Novartis Pharmaceutical Corporation, One Health Plaza, East Hanover, NJ, USA

3 Formerly Novartis Pharmaceutical Corporation, One Health Plaza, East Hanover, NJ, USA

4 Foundation for Sickle Cell Disease Research, 3858 Sheridan St, Suite S, Hollywood, FL, USA

\section{Introduction}

Sickle cell disease (SCD) is a hemoglobinopathy that causes red blood cells to lose their oxygen carrying capacity and is associated with severe, systemic vascular complications. It is estimated that approximately 100,000 Americans have SCD [1]. Patients with SCD experience chronic pain, cardiovascular events, ulcers, fatigue, organ damage, and sickle cellrelated pain crises, also referred to as vaso-occlusive crises (VOCs). Treatments used to manage symptoms or reduce complications of SCD include hydroxyurea, L-glutamine, and blood transfusions [2]; currently, the only available cure for SCD is bone marrow transplant [3]. 
Previous research has provided insight into many of the ways in which patients are impacted by SCD [4]. For example, patients with SCD report experiencing sleep disturbances [5], as well as deficits in both physical and mental well-being [6]. The extensive burden of SCD may also lead to an inability to maintain consistent work or schooling, engage in daily, social, or recreational activities, and participate in family life [7-10]. In addition to the burden of SCD, the experience of VOCs also has detrimental impacts on the lives of patients, though these impacts have been less comprehensively studied. VOCs are caused by multi-cell adhesion or cell clusters that block or reduce blood flow, and are a substantial cause of morbidity in patients with SCD; severe crises have also been associated with increased mortality [11]. These events are unpredictable and can cause disruption and hardship in the lives of patients, sometimes requiring medical attention in emergency departments or sickle cell urgent care centers, or leading to inpatient hospitalization [12]. Previous occurrence of VOCs has been linked to deficits in domains of health-related quality of life (HRQoL) such as general health, vitality, and bodily pain [6].

SCD is associated with high healthcare resource utilization (HCRU), with VOCs being the most common cause of hospital and emergency department visits among patients with SCD [13]. High rates of HCRU have been linked to a variety of poor outcomes among patients with $\mathrm{SCD}$, including lower HRQoL and likelihood of unemployment [14-17]. Despite high HCRU, especially for acute treatment of VOCs [18], the type of care patients with SCD receive may nevertheless be suboptimal. Receiving adequate treatment is a challenge commonly faced by patients with SCD, particularly for those who are transitioning from pediatric to adult care. Patients may have difficulty finding experienced practitioners to treat their SCD, coordinating communication between providers in a multi-disciplinary care setting, and obtaining or maintaining adequate insurance coverage, all of which could in turn result in over-reliance on emergency departments for treatment [19]. Perhaps unsurprisingly, patients themselves indicate that they prefer treatments they can administer at home [20], and report managing most of their VOCs at home [21]. Research has suggested that patients who prefer treating their pain at home believe that going to a hospital is not in their best interest and feel that they are responsible for managing their pain [22].

Given the seriousness of VOCs, there is an on-going need to better understand the ways in which these events impact patients with SCD. Although patients themselves have identified VOCs as one of the most debilitating aspects of their disease [20], minimal research has been conducted to quantify the extent of their impact on patients' lives. Therefore, additional research into their effect on patients' HRQoL and other outcomes is needed to provide insight into areas of unmet need and guide treatment development. Recognizing these gaps in the literature, the goal of this study was to examine the relationship between the frequency and severity of VOCs and HRQoL and work productivity impairment, using patient-reported data.

\section{Methods}

\section{Sample/study procedures}

The data for this analysis were drawn from an online, noninterventional, and cross-sectional study of adults with SCD. The study was approved by the New England Independent Review Board, and informed consent was obtained from all participants.

The patients were invited to participate in the study in 2018 through collaboration with patient advocacy groups and a market research company (Schlesinger Group). These groups distributed a description of the study, along with a hyperlink to the study's screening page, to potential participants. Patients who followed the hyperlink were directed to complete the screening survey. Patients were eligible to participate if they were aged $\geq 18$ years, self-reported having been diagnosed with SCD by a physician, currently resided in the US, and were willing to complete the online survey in English. Those who were deemed eligible to participate were automatically directed to complete the informed consent form, followed by the survey. Patients received the equivalent of a $\$ 75$ gift card for completing the survey. Data collection began on November 9, 2018, and ended on January $22,2019$.

\section{Study measures}

The online survey consisted of multiple modules designed to assess a variety of aspects of the patient experience, including demographic and disease characteristics; HRQoL; work productivity; VOC-related treatment experiences, HCRU, and management; barriers to receiving care; and impacts of SCD on employment, education, and personal relationships.

\section{Health-related quality of life}

The Adult Sickle Cell Quality of Life Measurement Information System (ASCQ-Me) is a disease-specific measure of HRQoL for patients with SCD [23]. The overall measurement system assesses 7 different health topics; 6 of these topics are assessed through 5-item questionnaires (Emotional Impact, Pain Impact, Sleep Impact, Social Functioning Impact, Stiffness Impact, Pain Episode), while the seventh topic is assessed through a 9-item questionnaire (SCD Medical History Checklist). The Pain Episode domain assesses the frequency of VOCs experienced in the past 12 months as 
well as the severity and degree of impact of the most recent VOC. Each of the 5 items in the Pain Episode domain can be used individually to describe the patient sample, or can be used together to create 2 composite scores: Pain Episode Frequency and Pain Episode Severity. This study used the static electronic forms to assess all domains except the SCD Medical History Checklist.

Each of the forms was scored according to developer guidelines and transformed to $t$ scores [23]. $T$ scores are standardized to have a mean of 50 and a standard deviation (SD) of 10, where a score of 50 represents the average SCD patient's HRQoL from a benchmark population of adults with SCD [23]. Higher domain scores represent a more favorable status for the Emotional, Pain, Sleep, Social Functioning, and Stiffness Impacts domains. Lower domain scores represent a more favorable status for the Pain Episode Frequency and Pain Episode Severity scores. The ASCQ-Me uses the phrase "pain attacks (crises)" to refer to VOCs and thus was adopted throughout the entirety of the online survey. This language was assessed during the original validation of the instrument, where it was determined that patients generally interpreted the phrase as the developers intended [23-26].

\section{Work productivity}

The Work Productivity and Activity Impairment: Specific Health Problem (WPAI:SHP) is a 6-item, self-report measure that assesses the impact of a person's specific health problem on work and daily non-work-related activities during the preceding week [27]. For this study, patients responded to each question in reference to their SCD.

The WPAI:SHP is scored to yield 4 domain scores. Amount of work time missed (absenteeism), impairment while at work (presenteeism), and overall productivity loss (absenteeism and presenteeism combined) are calculated for currently employed patients only. Activity impairment is calculated for all patients, regardless of current employment status, and reflects impairment in daily activities due to SCD. All WPAI:SHP domain scores are expressed as percentages, where larger values indicate greater impairment.

\section{VOC-related treatment experiences, healthcare resource utilization, and management}

Patients were presented with several questions related to treatment experiences and management of VOCs, including self-reported HCRU. Patients were asked to report the number of healthcare provider visits (not including visits to a hospital emergency room (ER), urgent care, or inpatient admission), the number of hospital ER or urgent care visits, and the number of hospital admissions they had in the past 12 months for treatment of VOCs. Patients were also asked to report where they typically receive treatment for VOCs. Those who reported managing at least $1 \mathrm{VOC}$ at home in the past 12 months were asked to report on the types of treatment they use and the reason they chose to treat their VOCs at home.

\section{Barriers to receiving treatment, and SCD-related impacts on employment, education, and personal relationships}

Patients were asked whether they had experienced any barriers to receiving SCD-related healthcare services. Response options were informed through literature review, a patient advisory board meeting, and clinician review. Patients were also asked to indicate whether SCD had ever impacted their employment status, education, or personal relationships. Patients who indicated that they had experienced negative impacts were asked to select from a list of specific negative outcomes which they had experienced (e.g., employment impacts: lost a job because of SCD, had to reduce work hours because of SCD; education impacts: dropped out of a school program because of SCD, did not enter a post-secondary school program because of SCD). For all barrier and impact-related items, patients could select more than one response option.

\section{Statistical analyses}

Descriptive statistics, such as frequencies and proportions (for categorical variables) and means, SDs, medians, and ranges (for continuous variables), were used to describe the sample in terms of patient characteristics, HRQoL scores, and WPAI scores. Descriptive statistics were also used to describe patients' treatment experiences and management of VOCs.

To examine the association between VOC frequency (or severity) and outcomes related to HRQoL and work impairment, a series of bivariate analyses were conducted.

Before the analyses were conducted, patients were stratified based on VOC frequency and severity. To assess VOC frequency, the first item of the ASCQ-Me Pain Episode domain was used; this item asks patients to report the number of VOCs they experienced in the past 12 months. Patients were stratified into one of 2 groups: those who experienced $0-3$ VOCs in the past 12 months and those who experienced $\geq 4$ VOCs in the past 12 months. This stratification was based on the distribution of data. To assess VOC severity, the Pain Episode Severity score of the ASCQ-Me was used. The items that comprise this score assess severity of pain during the patient's last VOC, the degree to which the last VOC interfered with the patient's life, and the duration of the patient's last VOC. As previously described, the ASCQ-Me domains are scored relative to an SCD benchmark mean of 50 and an SD of 10, with higher Pain Episode 
scores indicating less favorable status. Because there are currently no guidelines regarding what constitutes a "severe" or "less severe" VOC, the distribution of SCD benchmark scores was used to inform patient stratification. Specifically, patients whose ASCQ-Me Pain Episode Severity score was at least $1 / 2 \mathrm{SD}$ more severe than the SCD benchmark (i.e., scores $\geq 55$ ) were categorized as having "more severe" VOCs [28]. Patients whose ASCQ-Me Pain Episode Severity score was $<55$ were categorized as having "less severe" VOCs.

Kruskal-Wallis tests were conducted to explore the relationship between VOC frequency (or severity) and HRQoL and work productivity outcomes. Emotional, Social Functioning, Sleep, Stiffness, and Pain, as assessed by the ASCQ$\mathrm{Me}$, were used as the measures of HRQoL. In addition to testing for statistical significance between groups according to VOC frequency (or severity), average scores were compared to the SCD benchmark score of 50. Because no formal minimal importance difference (MID) has been established for the ASCQ-Me, $1 / 2$ SD of the average benchmark score (5 points), was used as the threshold to determine scores that differed meaningfully from the SCD benchmark score [28]. Absenteeism, presenteeism, overall productivity loss, and activity impairment, as assessed by the WPAI, were used as the measures of work productivity. Significance of all tests was assessed using an alpha level of 0.05 .

If a patient was missing data for a certain item, they were excluded from any analysis that used that item. All analyses were performed using SAS software version 9.4 (SAS Institute Inc.; Cary, NC, USA).

\section{Results}

\section{Analytic sample}

Of the 326 patients who completed the survey, 23 reported inconsistent responses across multiple survey items and thus were excluded from analysis. Inconsistent responses were defined either as (1) different responses to 2 items (one administered at screening and one administered later as part of the survey) regarding the number of VOCs experienced in the past 12 months in conjunction with a survey completion time of $<9$ min (completion time at or below the $25^{\text {th }}$ percentile among an interim sample of patients) or (2) inconsistent responses to items within the ASCQ-Me Pain Episode domain (e.g., reporting that they experienced 4 or more VOCs in the past 12 months [item 1 of the ASCQ-Me Pain Episode domain], followed by reporting that their last VOC was more than 5 years ago [item 2 of the ASCQ-Me Pain Episode domain]). The final analytic sample included 303 patients (Fig. 1).

\section{Sample characteristics}

The characteristics of the patient sample are depicted in Table 1 . The majority of patients were female and black or African American, and there was diversity in the patient sample in terms of educational attainment, type of health insurance, and US region of residence. The most frequently reported type of SCD within the sample was Hb-SS, followed by $\mathrm{Hb}$-SC. Patients reported receiving a variety of treatments for their SCD, including folic acid $(n=209,69.0 \%)$, opioid therapy $(n=176,58.1 \%)$, NSAIDS $(n=125,41.3 \%)$, hydroxyurea $(n=119,39.3 \%)$, IV fluids $(n=115,38.0 \%)$, and blood transfusions $(n=104,34.3 \%)$ (data not shown). Average ASCQ-Me scores were all within $1 \frac{1}{2}$ SD from the SCD benchmark score of 50, with SDs of 10 or less.

\section{VOC-related treatment experiences, healthcare resource utilization, and management}

\section{Treatment experiences}

As depicted in Table 2, the majority of patients had experienced at least $1 \mathrm{VOC}$ in the past 12 months ( $n=276,91.1 \%$ ); nearly half of the sample $(n=143,47.2 \%)$ experienced 4 or more VOCs during this time frame. Patients reported seeking treatment for VOCs at a variety of locations; the most frequently endorsed locations were the ER and at home.

\section{Home-managed VOCs}

Patients who had reported treating at least 1 VOC at home in the past 12 months reported using a variety of treatment methods including non-drug therapies, mind-body practices (such as meditation), non-narcotic analgesics, and mild narcotic analgesics. The majority of patients $(n=105,76.6 \%)$ reported that they treat their VOCs at home because they know how to treat their pain (Table 2).

\section{Healthcare resource utilization}

Average HCRU for VOCs among patients who experienced at least $1 \mathrm{VOC}$ in the past 12 months is also reported in Table 2. While some patients did not report using specific healthcare services for VOCs, the majority of the sample reported at least 1 healthcare visit in the past 12 months.

\section{Barriers to receiving treatment, and SCD-related impacts on employment, education, and personal relationships}

Approximately three-quarters of patients reported experiencing at least 1 barrier to receiving treatment for SCD (Table 3$) .59 .1 \%$ of patients $(n=179)$ reported that SCD 
Fig. 1 Flowchart of patient disposition. $A G$ advocacy groups, $M R C$ market research company
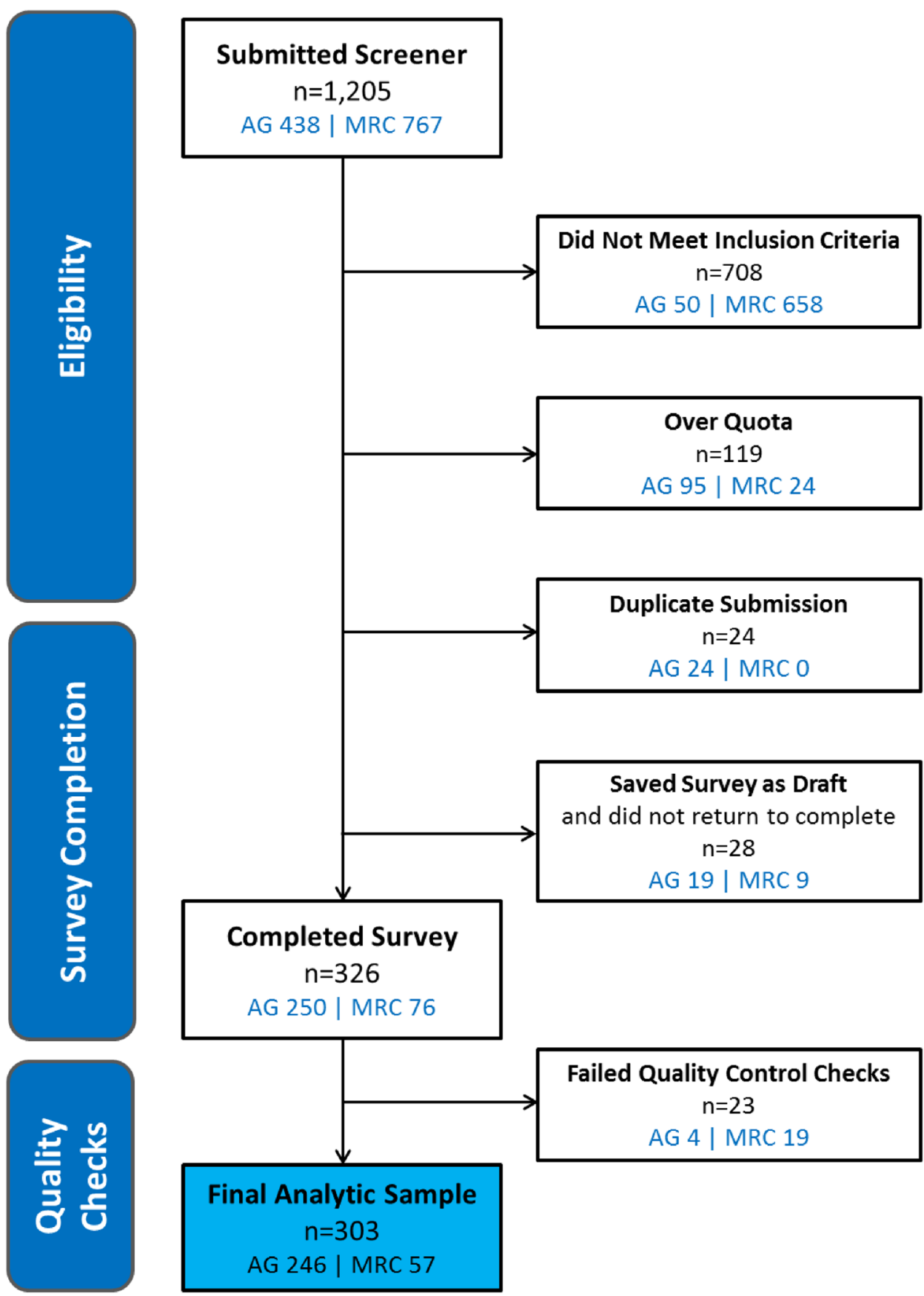

Duplicate Submission $\mathrm{n}=24$

AG 24 | MRC 0

Saved Survey as Draft

and did not return to complete $\mathrm{n}=28$

AG 19 | MRC 9
Failed Quality Control Checks $n=23$

AG 4 | MRC 19 had negatively impacted their employment status. Patients most frequently reported that they had to stop working ( $n=105,58.7 \%)$, take a leave of absence/unpaid time off $(n=94,52.5 \%)$, or reduce work hours $(n=89,49.7 \%)$ because of SCD.

$42.2 \%$ of patients $(n=128)$ reported that SCD had negatively impacted their education (Table 3 ). Patients most frequently reported that they had either delayed finishing a school program $(n=60,46.9 \%)$ or dropped out of a school program $(n=56,43.8 \%)$ because of SCD. Half of the patients $(n=153,50.5 \%)$ reported that SCD had negatively impacted their ability to start or continue a relationship.

\section{Health-related quality of life according to VOC frequency and severity}

Statistically significant differences in emotion, social functioning, stiffness, sleep, and pain were observed when stratifying patients according to the frequency of their VOCs over 
Table 1 Patient demographics, clinical characteristics, and health-related quality of life and work impairment scores
Demographic characteristics

Patients with $\operatorname{SCD}(N=303)$

$n$

Age, mean years (SD), median (IQR)

$34.37(10.25)$

$\%$

Gender

Female

$221 \quad 72.9$

Male

Non-binary/third gender

$81 \quad 26.7$

$1 \quad 0.3$

Race $^{\mathrm{a}}$

$\begin{array}{lll}\text { Black or African American } & 270 & 89.7\end{array}$

White $\quad 15 \quad 5.0$

$\begin{array}{lll}\text { Asian } & 2 & 0.7\end{array}$

American Indian or Alaska Native

$1 \quad 0.3$

Native Hawaiian or other Pacific Islander

$1 \quad 0.3$

Multiple races

2.0

Prefer not to answer

2.0

Education

Less than high school or some high school $\quad 18 \quad 6.0$

High school or equivalent (e.g., GED)

$39 \quad 12.9$

Some college, technical school, or associate's degree

$136 \quad 45.0$

$67 \quad 22.1$

$12 \quad 4.0$

Some graduate school but no degree

Graduate or professional degree (e.g., MBA, MS, MD, PhD)

$31 \quad 10.2$

Employment Status ${ }^{b}$

Currently employed (working for pay)

120

39.7

Unemployed

182

60.3

Health insurance ${ }^{c}$

Private insurance

$106 \quad 35.0$

Medicaid

$134 \quad 44.2$

Medicare

$103 \quad 34.0$

Veterans Health Insurance

0.7

Other (other type of insurance not listed, uninsured, or unsure)

2

37

12.2

US region of residence ${ }^{\mathrm{a}, \mathrm{d}}$

Northeast

$34 \quad 11.3$

South

$167 \quad 55.5$

Midwest

$66 \quad 21.9$

West

$34-11.3$

Type of $S^{b} D^{b}$

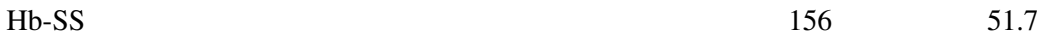

$\mathrm{Hb}-\mathrm{SC}$

$60 \quad 19.9$

$\mathrm{Hb}-\mathrm{S}$ beta ${ }^{+}$thalassemia

$20 \quad 6.6$

$\mathrm{Hb}-\mathrm{S}$ beta ${ }^{0}$ thalassemia

$22 \quad 7.3$

Other or do not know

$44 \quad 14.5$

Health-related quality of life

Mean (SD)

Median (IQR)

\section{ASCQ-Me}

Emotional impact

$46.57(8.00) \quad 46.20(41.20-51.50)$

Social functioning impact

$47.20(40.40-52.20)$

Stiffness impact

$47.00(9.21)$

$46.70(42.70-51.00)$

Sleep impact

$46.54(8.35)$

$\begin{array}{ll}48.71(6.54) & 48.20(45.00-53.90) \\ 47.87(9.48) & 47.10(41.50-54.00)\end{array}$

Pain impact

Pain episode frequency

$49.05(10.65) \quad 51.85(44.07-55.73)$

Pain episode severity 
Table 1 (continued)

\begin{tabular}{lll}
\hline Work impairment & Mean (SD) & Median (IQR) \\
\hline WPAI:SHP & & \\
$\quad$ Absenteeism & $27.54(29.04)$ & $20.00(0.00-50.00)$ \\
Presenteeism & $47.19(30.50)$ & $50.00(20.00-70.00)$ \\
$\quad$ Overall work productivity & $55.19(33.28)$ & $61.51(28.89-85.00)$ \\
$\quad$ loss & & $60.00(30.00-80.00)$ \\
$\quad$ Overall activity impairment & $53.15(28.88)$ & \\
\hline
\end{tabular}

ASCQ-Me Adult Sickle Cell Quality of Life Measurement Information System; WPAI:SHP Work Productivity and Activity Impairment: Specific Health Problem, $S C D$ sickle cell disease, $S D$ standard deviation, $I Q R$ inter-quartile range

${ }^{a}$ Data from 2 patients are missing; frequency based on available data $(N=301)$

${ }^{\mathrm{b}}$ Data from 1 patient is missing; frequency based on available data $(N=302)$

${ }^{c}$ Multiple response options allowed; frequency sums to $>100 \%$

${ }^{\mathrm{d}}$ Regions defined according to US Census Bureau

${ }^{\mathrm{e}}$ Higher ASCQ-Me impact scores indicate better functioning. Higher ASCQ-Me pain episode scores indicate worse functioning

${ }^{\mathrm{f}}$ Higher WPAI:SHP scores indicate greater impairment. Absenteeism scores were calculated for patients who were employed at the time of the survey $(N=118)$. Presenteeism and overall work productivity scores were calculated for patients who were both employed and reported working in the past 7 days $(N=114)$. Overall activity impairment scores calculated for all patients $(N=302$; data from 1 patient is missing)

the past 12 months (Table 4; Panel a of Fig. 2). Patients with more frequent VOCs reported worse HRQoL across all of these domains, as measured by the ASCQ-Me, than patients with less frequent VOCs. Patients who experienced 0-3 VOCs in the past 12 months reported HRQoL scores that were equivalent to the SCD benchmark score of 50, meaning that their scores were similar to that of the average SCD patient, as defined by the benchmark sample. However, patients who experienced $\geq 4$ VOCs in the past 12 months reported emotion, social functioning, and stiffness impacts that were $1 / 2$ SD worse than the SCD benchmark.

An additional set of analyses was conducted to explore HRQoL according to different stratifications of VOC frequency. Specifically, patients were divided into 3 groups: 0 VOCs, 1 VOC, and $\geq 2$ VOCs. The overall pattern of results remained unchanged, as patients with $\geq 2$ VOCs in the past 12 months had lower (i.e.,worse) HRQoL scores than patients with 0 or 1 VOCs (data not shown). Due to the limited number of patients who experienced either 0 or 1 VOC in the past 12 months, however, this stratification was not pursued for the other outcomes of interest (i.e., work impairment).

Statistically significant differences in emotion, social functioning, stiffness, sleep, and pain were also observed when stratifying patients according to the severity of their last VOC (Table 5; Panel b of Fig. 2). Patients with less severe VOCs reported mean scores across all domains that were similar to the SCD benchmark score; patients with more severe VOCs reported mean emotion, social functioning, and stiffness scores that were $1 / 2$ SD worse than the SCD benchmark.

\section{Work productivity according to VOC frequency and severity}

Statistically significant differences in 3 of the 4 WPAI scores were observed when patients were stratified by VOC frequency (Table 6; Panel a of Fig. 3). Specifically, patients with $\geq 4$ VOCs in the past 12 months reported greater absenteeism, overall work productivity loss, and activity loss than patients with $0-3$ VOCs $(P<0.05$ for all $)$; presenteeism did not differ significantly according to VOC frequency.

A similar pattern of results was observed when stratifying patients by VOC severity. Patients with more severe VOCs reported greater absenteeism, overall work productivity loss, and activity impairment in the 7 days preceding survey administration than patients with less severe VOCs $(P<0.05$ for all) (Table 7; Panel b of Fig. 3).

\section{Discussion}

The goal of this study was to explore the disease experience of patients with SCD, focusing primarily on better understanding the ways in which patients are impacted by the frequency and severity of VOCs. To our knowledge, this is one of the few studies designed to comprehensively investigate the impacts of both SCD and VOCs on multiple dimensions of patients' lives. Data obtained from the cross-sectional online survey of adults with SCD provided evidence to demonstrate that patients experience impacts of SCD across many different aspects of their lives, including 
Table 2 Treatment experiences and management of VOCs

Patients with SCD $(N=303)$

n \%

Number of VOCs experienced in the past 12 months

1

27

29

8.9

2

$44 \quad 14.5$

3

60

19.8

4 or more

Location at which patients typically receive treatment for $\mathrm{VOCs}^{\mathrm{a}, \mathrm{b}}$

Home

Primary care doctor's office

$54 \quad 18.4$

Hematologist's office

$108 \quad 36.7$

Specialized SCD center, acute care center, or day clinic

$63 \quad 21.4$

Hospital outpatient clinic

$67 \quad 22.8$

Hospital inpatient setting

ER or urgent care

Treatments patients use to manage VOCs at home ${ }^{\mathrm{a}, \mathrm{c}}$

Non-narcotic analgesics (e.g., Tylenol, aspirin, Advil)

Mild narcotic analgesics/opioids (e.g., codeine, oxycodone)

Strong narcotic analgesics/opioids (e.g., morphine, hydromorphone, meperidine)

Herbal medicines

Homeopathic remedies

Mind/body practices (e.g., meditation, relaxation techniques, yoga)

Other non-drug therapies (e.g., rest, fluids, heating pad)

Other

Reasons patients treat their VOCs at home $\mathrm{a}^{\mathrm{a}, \mathrm{c}}$

$\begin{array}{ll}\text { I know what to do to treat my pain } & 105\end{array}$

76.6

My pain is mild

$49 \quad 35.8$

I do not consider the need to go elsewhere to treat my pain attacks (crises)

I have limited or no access to other treatment options

$19 \quad 13.9$

I do not think others are able or willing to treat my pain attacks (crises)

It is difficult to find transportation to receive treatment elsewhere

18

13.1

It is too expensive to receive treatment elsewhere

\begin{tabular}{|c|c|c|}
\hline It is too expensive to receive treatment elsewhere & 25 & 18.2 \\
\hline & Mean (SD) & Median (range) \\
\hline \multicolumn{3}{|l|}{ HCRU for VOCs in the past 12 months } \\
\hline Number of visits to a health provider for treatment of $\mathrm{VOCs}^{\mathrm{d}}$ & $4.78(6.11)$ & $3.00(0-48)$ \\
\hline Number of visits to a hospital ER and/or urgent care for treatment of VOCs ${ }^{d}$ & $5.27(10.34)$ & $3.00(0-100)$ \\
\hline Number of overnight or longer hospital stays for treatment of VOCs ${ }^{\mathrm{d}}$ & $4.17(8.81)$ & $2.00(0-100)$ \\
\hline $\begin{array}{l}\text { Number of nights spent in hospital during each overnight or longer hospital stay for treat- } \\
\text { ment of VOCs }\end{array}$ & $6.73(10.00)$ & $4.00(1-100)$ \\
\hline
\end{tabular}

$E R$ emergency room, $S C D$ sickle cell disease, $S D$ standard deviation, VOCs vaso-occlusive crises, HCRU Healthcare resource utilization

${ }^{a}$ Multiple response options allowed; frequency sums to $>100 \%$

${ }^{\mathrm{b}}$ Items administered only to patients who indicated they have experienced at least one VOC in their lifetime $(N=294)$

${ }^{\mathrm{c}}$ Item administered only to patients who have managed at least one VOC at home in the past 12 months $(N=137)$

${ }^{\mathrm{d}}$ Item administered only to patients who indicated they have experienced at least one VOC in the past 12 months $(N=276)$

${ }^{\mathrm{e}}$ Item administered only to patients who indicated they were hospitalized overnight for VOCs in the past 12 months $(N=215)$ 
Table 3 Barriers to care and impacts on employment, education, and personal relationships due to SCD

Patients with $\operatorname{SCD}(N=303)$

$n$

Barriers patients have experienced to receiving healthcare for $\mathrm{SCD}^{\mathrm{a}}$

Difficulty affording healthcare services $\quad 89$

29.4

Limited or lack of health insurance

26.4

Difficulty obtaining transportation to receive healthcare services

16.8

Discrimination or stigmatization by healthcare professionals

39.6

Difficulty trusting healthcare professionals

33.3

Limited or lack of specialized SCD centers, acute care centers, or day clinics

Other

None of the above

Impact on employment ${ }^{\mathrm{a}}$

$\begin{array}{lr}\text { Yes, it has positively impacted my employment status } & 31\end{array}$

10.2

Yes, it has negatively impacted my employment status

59.1

No, it has not impacted my employment status

Not applicable

Type of negative employment impacts ${ }^{\mathrm{a}, \mathrm{b}}$

Lost a job because of SCD

Stopped working because of SCD

Changed jobs/professions because of SCD

30.2

Reduced work hours because of SCD

49.7

Took a leave of absence or unpaid time off because of SCD

Changed job responsibilities because of SCD

Have not sought a promotion, or been granted a promotion because of SCD

Other

Impact on education $^{\mathrm{a}}$

Yes, it has positively impacted my level of education

Yes, it has negatively impacted my level of education

No, it has not impacted my level of education

I do not know / I am not sure

Type of negative education impacts ${ }^{\mathrm{a}, \mathrm{c}}$

Did not enter a post-secondary school program (e.g., college or technical school) because of SCD

Changed area of study because of SCD

Delayed beginning a school program because of SCD

Delayed finishing a school program because of SCD

Dropped out of a school program because of SCD

Other

Negative impact on personal relationships

Yes

No

I do not know/I am not sure

Not applicable

$S C D$ sickle cell disease

${ }^{a}$ Multiple response options allowed; frequency sums to $>100 \%$

${ }^{\mathrm{b}}$ Items administered only to patients who indicated experiencing a negative impact on employment $(N=179)$

${ }^{\mathrm{c}}$ Items administered only to patients who indicated experiencing a negative impact on education $(N=128)$ 
Table 4 HRQoL according to VOC frequency

\begin{tabular}{|c|c|c|c|c|c|c|c|c|c|c|c|}
\hline \multirow[t]{2}{*}{ ASCQ-Me domain ${ }^{\mathrm{a}}$} & \multicolumn{5}{|c|}{$0-3$ VOCs in the past 12 months } & \multicolumn{5}{|c|}{$\geq 4$ VOCs in the past 12 months } & \multirow[t]{2}{*}{$P^{\mathrm{b}}$} \\
\hline & $N$ & Mean & $\mathrm{SD}$ & Median & IQR & $N$ & Mean & $\mathrm{SD}$ & Median & IQR & \\
\hline Emotional impact & 160 & 48.16 & 8.33 & 47.40 & $42.50-54.25$ & 143 & 44.80 & 7.23 & 44.90 & $39.90-48.70$ & 0.001 \\
\hline Social functioning impact & 160 & 49.92 & 9.48 & 48.80 & $43.90-55.80$ & 143 & 43.73 & 7.71 & 43.90 & $38.70-48.80$ & $<0.001$ \\
\hline Stiffness impact & 160 & 48.20 & 8.72 & 48.10 & $42.70-52.70$ & 143 & 44.67 & 7.52 & 45.40 & $39.90-49.50$ & 0.001 \\
\hline Sleep impact & 160 & 49.87 & 6.65 & 49.70 & $45.00-55.30$ & 143 & 47.42 & 6.19 & 46.70 & $43.20-51.10$ & $<0.001$ \\
\hline Pain impact & 160 & 50.06 & 9.49 & 48.50 & $44.40-58.00$ & 143 & 45.41 & 8.89 & 45.70 & $38.30-51.20$ & $<0.001$ \\
\hline
\end{tabular}

ASCQ-Me Adult Sickle Cell Quality of Life Measurement Information System, VOCs vaso-occlusive crises, SD standard deviation, IQR interquartile range, HRQoL Health-related quality of life

${ }^{a}$ Higher ASCQ-Me impact scores indicate better functioning

${ }^{\mathrm{b}}$ After Bonferroni correction, all associations remained statistically significant, with $P<$ the Bonferroni correction-adjusted critical value of 0.01

Fig. 2 HRQoL according to VOC frequency (a) and severity (b). HRQoL scores differ as a function of VOC frequency/ severity for all domains $(P<0.05)$. Higher ASCQ-Me impact scores indicate better functioning. Less severe VOCs = ASCQ-Me Pain Episode Severity score $<55$; more severe VOCs = ASCQ-Me Pain Episode Severity score $\geq 55$. ASCQ-Me Adult Sickle Cell Quality of Life Measurement Information System, HRQoL health-related quality of life, $S C D$ sickle cell disease, $V O C s$ vaso-occlusive crises
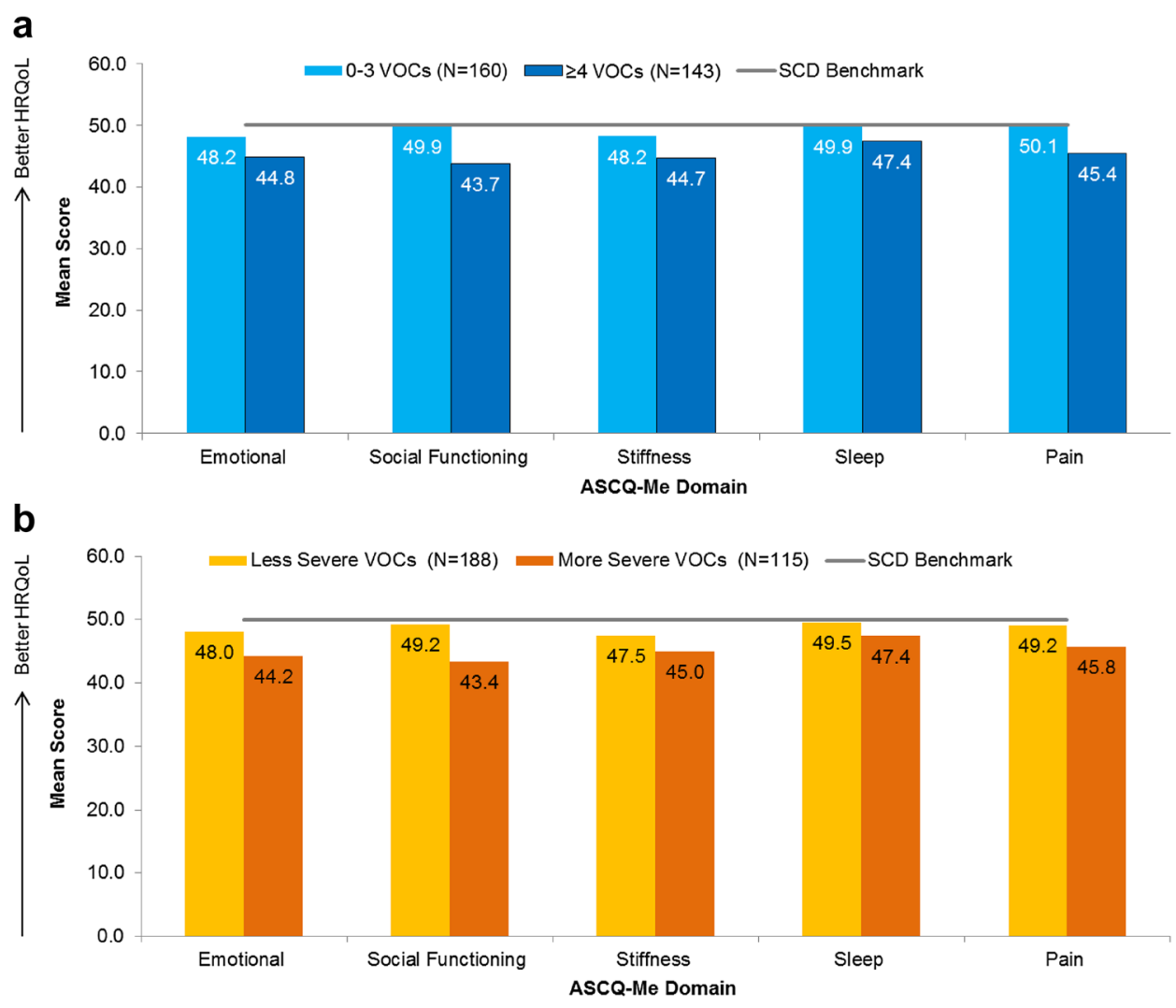

employment, education, and personal relationships. In addition, the frequency and severity of VOCs were associated with impacts on HRQoL and work productivity.

The frequency and severity of VOCs were related to multiple domains of HRQoL. In particular, patients with more frequent (or severe) VOCs reported greater impacts on areas known to be affected by SCD, as measured by the ASCQ-Me, than patients with less frequent (or severe) VOCs. Moreover, these patients experienced deficits in areas of emotional functioning, social functioning, and stiffness that were meaningfully lower than a benchmark SCD population. These findings extend past research that has linked the previous occurrence of VOCs to lower scores on general health, vitality, and bodily pain domains (as measured by the SF-36® Health Survey) [6] by demonstrating that HRQoL is impacted not only by any past experience of VOCs, but also by the frequency and severity of these experiences. The findings of the current study also highlight impacts that extend beyond physical functioning, illustrating the effects of VOCs on social and emotional functioning as well.

In addition to impacts on HRQoL, results demonstrate that the frequency of VOCs is associated with specific aspects of work productivity. The number of VOCs over a 12-month period was related to the amount of missed worktime in the 
Table 5 HRQoL according to VOC severity

\begin{tabular}{|c|c|c|c|c|c|c|c|c|c|c|c|}
\hline \multirow[t]{2}{*}{ ASCQ-Me domain $^{\mathrm{a}}$} & \multicolumn{5}{|c|}{ Less severe VOCs } & \multicolumn{5}{|c|}{ More severe VOCs } & \multirow[t]{2}{*}{$P^{\mathrm{b}}$} \\
\hline & $N$ & Mean & $\mathrm{SD}$ & Median & IQR & $N$ & Mean & SD & Median & IQR & \\
\hline Emotional impact & 188 & 48.00 & 7.36 & 47.40 & $43.10-51.50$ & 115 & 44.24 & 8.48 & 43.70 & $39.90-48.70$ & $<0.001$ \\
\hline Social functioning impact & 188 & 49.22 & 8.61 & 48.80 & $43.90-54.00$ & 115 & 43.37 & 9.05 & 42.10 & $36.80-47.20$ & $<0.001$ \\
\hline Stiffness impact & 188 & 47.49 & 7.80 & 46.70 & $44.00-51.00$ & 115 & 44.97 & 8.99 & 45.40 & $38.40-49.50$ & 0.008 \\
\hline Sleep impact & 188 & 49.52 & 6.58 & 49.70 & $45.00-53.90$ & 115 & 47.40 & 6.29 & 46.70 & $43.20-51.10$ & 0.005 \\
\hline Pain impact & 188 & 49.16 & 8.93 & 47.10 & $44.40-55.80$ & 115 & 45.75 & 10.00 & 44.40 & $38.30-51.20$ & 0.002 \\
\hline
\end{tabular}

More severe VOCs: ASCQ-Me Pain Episode Severity score $\geq 55$ (1/2 SD worse than the benchmark score); less severe VOCs: ASCQ-Me Pain Episode Severity score $<55$

$A S C Q-M e$ Adult Sickle Cell Quality of Life Measurement Information System, VOCs vaso-occlusive crises, SD standard deviation, IQR interquartile range, HRQoL Health-related quality of life

${ }^{a}$ Higher ASCQ-Me impact scores indicate better functioning

${ }^{\mathrm{b}}$ After Bonferroni correction, all associations remained statistically significant, with $P<$ the Bonferroni correction-adjusted critical value of 0.01

Table 6 Work productivity according to VOC frequency

\begin{tabular}{|c|c|c|c|c|c|c|c|c|c|c|c|}
\hline \multirow[t]{2}{*}{ WPAI:SHP domain ${ }^{a}$} & \multicolumn{5}{|c|}{$0-3$ VOCs in the past 12 months } & \multicolumn{5}{|c|}{$\geq 4$ VOCs in the past 12 months } & \multirow[t]{2}{*}{$P^{\mathrm{b}}$} \\
\hline & $N$ & Mean & SD & Median & IQR & $N$ & Mean & SD & Median & IQR & \\
\hline Absenteeism & 67 & 21.87 & 26.25 & 14.29 & $0.00-36.84$ & 51 & 34.99 & 31.06 & 30.77 & $0.00-57.14$ & 0.018 \\
\hline Presenteeism & 65 & 42.77 & 30.49 & 50.00 & $10.00-70.00$ & 49 & 53.06 & 29.81 & 60.00 & $30.00-80.00$ & 0.068 \\
\hline Overall work productivity loss & 65 & 49.27 & 34.01 & 55.00 & $10.00-81.05$ & 49 & 63.03 & 30.90 & 70.00 & $30.00-88.89$ & 0.016 \\
\hline Overall activity impairment & 160 & 48.25 & 29.96 & 50.00 & $20.00-70.00$ & 142 & 58.66 & 26.66 & 60.00 & $40.00-80.00$ & 0.003 \\
\hline
\end{tabular}

WPAI:SHP Work Productivity and Activity Impairment: Specific Health Problem, VOCs vaso-occlusive crises, $S D$ standard deviation, IQR interquartile range

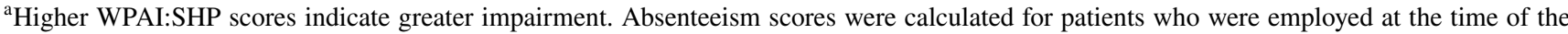
survey $(N=118)$. Presenteeism and overall work productivity scores were calculated for patients who were both employed and reported working in the past $7(N=114)$. Overall activity impairment scores calculated for all patients $(N=302$; data from 1 patient is missing)

${ }^{\mathrm{b}}$ After Bonferroni correction, the association for overall activity impairment remained statistically significant, with $P<$ the Bonferroni correction-adjusted critical value of 0.0125

7 days preceding survey administration, suggesting that the impacts of VOCs on patients' lives may extend beyond the end of the event itself. To the best of our knowledge, the WPAI has not been administered to patients with SCD in an observational setting. As such, the results obtained from this study cannot be compared to, or interpreted, in light of previous findings. However, findings can be compared to those reported in different disease areas, helping to better contextualize the current results. Specifically, the quantity of absenteeism reported by patients with the most frequent or severe VOCs is nearly double what has been reported by patients with non-malignant chronic pain (19.4\%) [29], and by patients who have recently completed treatment for breast cancer (21-25\%) [30], but slightly less than the absenteeism reported by patients who currently have breast cancer (56-61\%) [30]. Absenteeism is calculated as the number of work hours missed, divided by the total number of hours a patient could have worked. Thus, an absenteeism score of 34.99 (the average score of patients with the most frequent VOCs) is generally equivalent to missing
$14 \mathrm{~h}$ of a 40-h work week, while an absenteeism score of 39.59 (the average score of patients with the most severe VOCs) is generally equivalent to missing $16 \mathrm{~h}$ of a 40 -h week. Put this way, the impacts of VOCs can be described more concretely, elucidating the ways in which employed patients with frequent or severe VOCs are impacted by SCD.

Given the degree of work impairment experienced by patients with SCD, and in particular by those with more frequent or severe VOCs, it is unsurprising that many patients also reported experiencing negative impacts on their overall employment status. Previous qualitative work has reported that patients with SCD find it difficult to manage their jobs. For example, the FDA's Voice of the Patient report describes that patients with SCD experience difficulty keeping up with their work due to both absences from work and stress caused by various aspects of the disease [20]. Other qualitative research has documented patients' descriptions of challenges related to finding and maintaining adequate employment; patients discussed difficulty keeping jobs or building job 
Fig. 3 Work impairment according to VOC frequency (a) and severity (b). *WPAI:SHP domain scores differed as a function VOC frequency/ severity $(P<0.05)$. Higher WPAI:SHP scores indicate greater impairment. For sample sizes for per VOC category and WPAI:SHP domain, refer to Tables 6 and 7. Less severe VOCs $=$ ASCQ-Me Pain Episode Severity score $<55$; more severe VOCs = ASCQ-Me Pain Episode Severity score $\geq 55$. WPAI:SHP Work Productivity and Activity Impairment: Specific Health Problem, VOCs vaso-occlusive crises

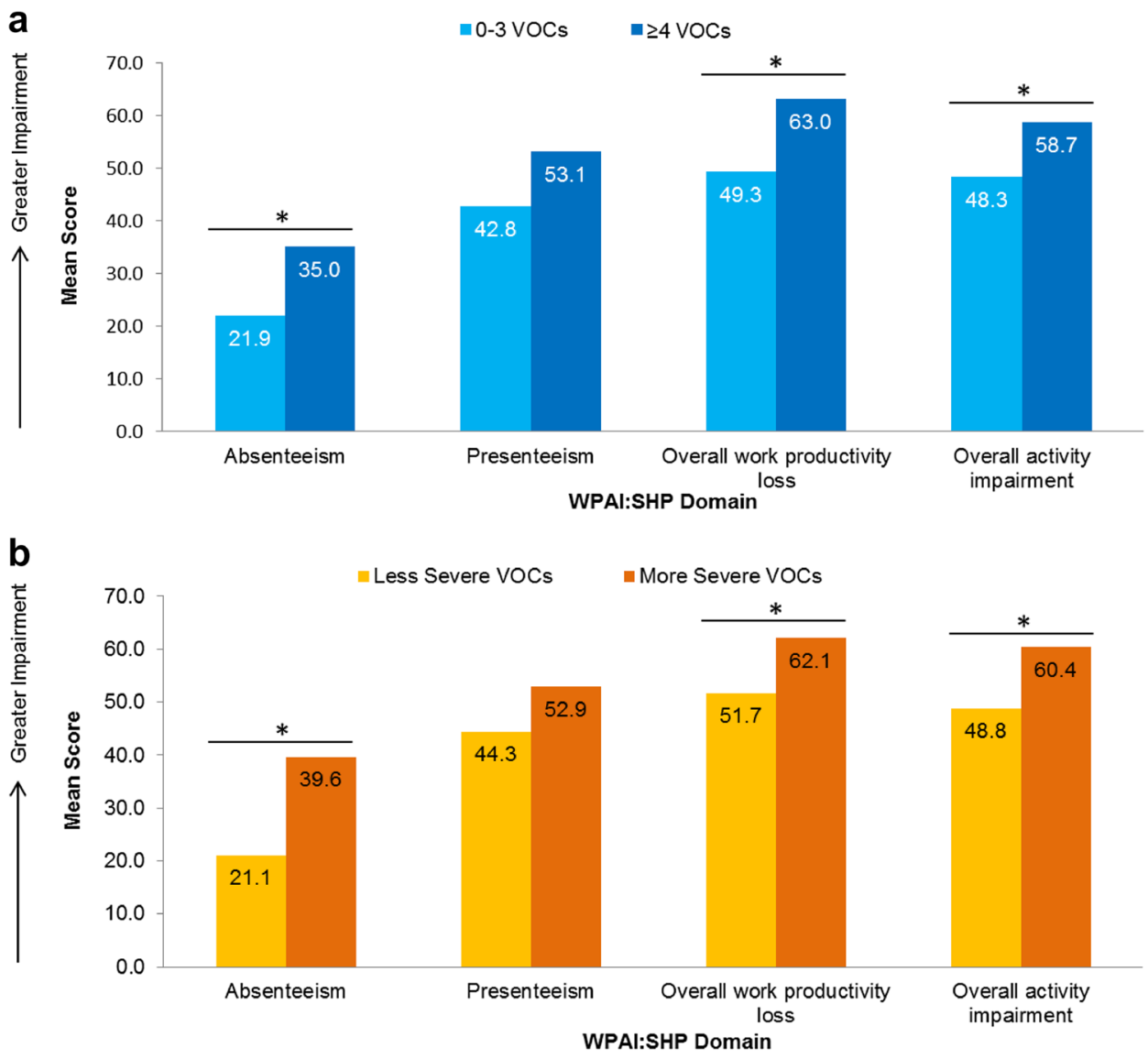

Table 7 Work productivity according to VOC severity

\begin{tabular}{|c|c|c|c|c|c|c|c|c|c|c|c|}
\hline \multirow[t]{2}{*}{ WPAI:SHP domain ${ }^{a}$} & \multicolumn{5}{|c|}{ Less severe VOCs } & \multicolumn{5}{|c|}{ More severe VOCs } & \multirow[t]{2}{*}{$P^{\mathrm{c}}$} \\
\hline & $N^{\mathrm{b}}$ & Mean & SD & Median & IQR & $N$ & Mean & SD & Median & IQR & \\
\hline Absenteeism & 77 & 21.12 & 23.91 & 16.67 & $0.00-37.50$ & 41 & 39.59 & 33.96 & 30.77 & $10.00-62.50$ & 0.003 \\
\hline Presenteeism & 76 & 44.34 & 28.49 & 50.00 & $20.00-70.00$ & 38 & 52.89 & 33.84 & 60.00 & $20.00-80.00$ & 0.122 \\
\hline Overall work productivity loss & 76 & 51.73 & 31.50 & 57.39 & $29.44-78.57$ & 38 & 62.10 & 36.02 & 76.60 & $27.42-91.67$ & 0.043 \\
\hline Overall activity impairment & 188 & 48.78 & 27.77 & 50.00 & $30.00-70.00$ & 114 & 60.35 & 29.36 & 70.00 & $40.00-80.00$ & 0.001 \\
\hline
\end{tabular}

WPAI:SHP Work Productivity and Activity Impairment: Specific Health Problem, VOCs vaso-occlusive crises, $S D$ standard deviation, IQR interquartile range

${ }^{a}$ Higher WPAI:SHP scores indicate greater impairment. Absenteeism scores were calculated for patients who were employed at the time of the survey $(N=118)$. Presenteeism and overall work productivity scores were calculated for patients who were both employed and reported working in the past $7(N=114)$. Overall activity impairment scores calculated for all patients $(N=302$; data from 1 patient is missing)

${ }^{b}$ More severe VOCs: ASCQ-Me Pain Episode Severity score $\geq 55$ ( $1 \frac{1}{2}$ SD worse than the benchmark score); less severe VOCs: ASCQ-Me Pain Episode Severity score $<55$

${ }^{\mathrm{c}}$ After Bonferroni correction, the association for absenteeism and overall activity impairment remained statistically significant, with $P<$ the Bonferroni correction-adjusted critical value of 0.0125

history, and having to leave jobs that were too physically demanding [10]. Our results extend these findings by helping to quantify the frequency with which patients experience such impacts, showing that such experiences are relatively widespread among patients with SCD. Similar to the impacts on employment, patients in our study also reported that their SCD had impacted their personal relationships and negatively impacted their education. Overall, the number of patients who experienced negative impacts on education was fewer than those who experienced negative impacts on employment. Patients with SCD often experience a difficult transition from pediatric to adult care [31], and may struggle to obtain consistent and effective care as young adults, thus potentially increasing the likelihood that SCD will 
negatively affect various facets of their adult lives. Indeed, approximately $75 \%$ of the patients in this study reported experiencing barriers to receiving care for their SCD, such as difficulty affording healthcare services, limited health insurance, discrimination by healthcare professionals, difficulty trusting healthcare professionals, and a lack of specialized treatment centers. Increasing access, options, and quality of SCD-related care may improve patients' employment-related outcomes.

This study had some limitations. As with any information collected through patient report, recall bias could affect reports of events. Second, diagnosis of SCD was entirely self-reported. While recruitment of patients through collaboration with SCD-related organizations and advocacy groups in the absence of explicit physician confirmation has been reported elsewhere [32, 33], the trade-offs between relying on self-report (e.g., more expedient data collection, ability to recruit across a broad geographic region) and obtaining additional confirmation must be considered. Third, selection bias could affect the type of patients who participated in the survey; the survey could only be completed by individuals with internet access, and those who are unfamiliar or less comfortable using this type of technology may have been less likely to participate. Fourth, the study was designed to be cross-sectional, exploratory, and largely descriptive. As such, none of the relationships reported here can be interpreted as causal, nor can longitudinal relationships be inferred. However, the results of the study are informative in their own right and can provide a solid foundation for additional future research.

Balancing the aforementioned limitations, this study also had several particular strengths. First, the study sample was quite large, particularly for a rare disease. Second, evaluation of the study sample strongly suggests that it is generally representative of the larger SCD patient population. While more women than men completed the survey, scores obtained on the ASCQ-Me were nearly identical to those from an SCD benchmark population [23], and the distribution of patients across race, types of SCD, and across US geographic regions is similar to what has been reported in previous studies [34]. Third, the survey assessed a variety of different concepts related to the experience of patients with SCD, including both validated patient-reported outcome measures and items written specifically for this study. This approach allowed for a clearer assessment of the ways in which patients are impacted by the disease. For example, only relying on the WPAI to measure work-related outcomes would capture the experiences only of patients who were currently employed, failing to take into consideration the perspectives of unemployed patients, who comprised $61 \%$ of the total study sample. Rather, the WPAI was fielded in conjunction with a series of items regarding the lifetime experiences of all patients, regardless of current employment status, thus providing a more complete understanding of this particular outcome.

This study provides evidence to demonstrate a link between patient outcomes such as HRQoL and work impairment, and the frequency and severity of VOCs. The findings presented in this study provide a solid foundation for future research, which should aim to investigate a causal relationship between these factors. Additional research should also explore how health interventions or the alleviation of structural or environmental barriers to receiving healthcare may improve HRQoL and employment opportunities among patients with SCD.

In conclusion, this study provides a comprehensive description of the patient experience with SCD, with a specific emphasis on highlighting the ways in which VOC frequency and severity impact patients' HRQoL and work productivity. This research provides evidence to suggest that VOCs may have broad and cumulative impact on aspects of life such as emotional and social functioning which may last beyond the end of the event itself.

Funding This study was funded by Novartis Pharmaceuticals Corporation.

\section{Compliance with ethical standards}

Conflict of interest AAR, XL, KLM, and MKW are full-time employees of Optum and received research funding from Novartis Pharmaceuticals Corporation to conduct the study. MB, JP, and SN are full-time employees of the study sponsor, Novartis Pharmaceuticals Corporation; MB and JP also own stock in the company. LBH has received funding from the study sponsor, Novartis Pharmaceuticals Corporation, and from Bluebird Bio and Pfizer Inc. RH has nothing to disclose.

Ethical approval All procedures performed in studies involving human participants were in accordance with the ethical standards of the institutional and or national research committee and with the 1964 Helsinki Declaration and its later amendments or comparable ethical standards.

Informed consent The informed consent form, protocol, and recruitment materials were approved by the New England Independent Review Board (IRB \# 120180240). All participants provided consent.

Open Access This article is licensed under a Creative Commons Attribution 4.0 International License, which permits use, sharing, adaptation, distribution and reproduction in any medium or format, as long as you give appropriate credit to the original author(s) and the source, provide a link to the Creative Commons licence, and indicate if changes were made. The images or other third party material in this article are included in the article's Creative Commons licence, unless indicated otherwise in a credit line to the material. If material is not included in the article's Creative Commons licence and your intended use is not permitted by statutory regulation or exceeds the permitted use, you will need to obtain permission directly from the copyright holder. To view a copy of this licence, visit http://creativecommons.org/licenses/by/4.0/ 


\section{References}

1. Centers for Disease Control and Prevention. (2017). Sickle cell disease. Retrieved 11 April 2019, from https://www.cdc.gov/ ncbddd/sicklecell/treatments.html.

2. National Heart, Lung, and Blood Institute. (2014). Evidence-based management of sickle cell disease: Expert panel report. https:// www.nhlbi.nih.gov/health-topics/all-publications-and-resources/ evidence-based-management-sickle-cell-disease-expert-0.

3. Bolaños-Meade, J., \& Brodsky, R. A. (2009). Blood and marrow transplantation for sickle cell disease: Overcoming barriers to success. Current Opinion in Oncology. https://doi.org/10.1097/ CCO.0b013e328324ba04.

4. Panepinto, J. A., \& Bonner, M. (2012). Health-related quality of life in sickle cell disease: Past, present, and future. Pediatric Blood \& Cancer. https://doi.org/10.1002/pbc.24176.

5. Wallen, G. R., Minniti, C. P., Krumlauf, M., Eckes, E., Allen, D., Oguhebe, A., et al. (2014). Sleep disturbance, depression and pain in adults with sickle cell disease. BMC Psychiatry. https://doi. org/10.1186/1471-244X-14-207.

6. Dampier, C., LeBeau, P., Rhee, S., Lieff, S., Kesler, K., Ballas, S., et al. (2011). Health-related quality of life in adults with sickle cell disease (SCD): A report from the comprehensive sickle cell centers clinical trial consortium. American Journal of Hematology. https:// doi.org/10.1002/ajh.21905.

7. Fuggle, P., Shand, P. A., Gill, L. J., \& Davies, S. C. (1996). Pain, quality of life, and coping in sickle cell disease. Archives of Disease in Childhood, 75(3), 199-203.

8. Badawy, S. M., Thompson, A. A., Lai, J.-S., Penedo, F. J., Rychlik, K., \& Liem, R. I. (2017). Adherence to hydroxyurea, health-related quality of life domains, and patients' perceptions of sickle cell disease and hydroxyurea: A cross-sectional study in adolescents and young adults. Health and Quality of Life Outcomes. https://doi. org/10.1186/s12955-017-0713-x.

9. Badawy, S. M., Thompson, A. A., Penedo, F. J., Lai, J.-S., Rychlik, K., \& Liem, R. I. (2017). Barriers to hydroxyurea adherence and health-related quality of life in adolescents and young adults with sickle cell disease. European Journal of Haematology. https://doi. org/10.1111/ejh.12878.

10. Matthie, N., Hamilton, J., Wells, D., \& Jenerette, C. (2016). Perceptions of young adults with sickle cell disease concerning their disease experience. Journal of Advanced Nursing. https://doi. org/10.1111/jan.12760.

11. Darbari, D. S., Wang, Z., Kwak, M., Hildesheim, M., Nichols, J., Allen, D., et al. (2013). Severe painful vaso-occlusive crises and mortality in a contemporary adult sickle cell anemia cohort study. PLoS ONE. https://doi.org/10.1371/journal.pone.0079923.

12. Brousseau, D. C., Owens, P. L., Mosso, A. L., Panepinto, J. A., \& Steiner, C. A. (2010). Acute care utilization and rehospitalizations for sickle cell disease. JAMA . https://doi.org/10.1001/ jama.2010.378.

13. Ballas, S. K., \& Lusardi, M. (2005). Hospital readmission for adult acute sickle cell painful episodes: Frequency, etiology, and prognostic significance. American Journal of Hematology. https://doi. org/10.1002/ajh.20336.

14. Carroll, C. P., Cichowitz, C., Yu, T., Olagbaju, Y. O., Nelson, J. A., Campbell, T., et al. (2018). Predictors of acute care utilization and acute pain treatment outcomes in adults with sickle cell disease: The role of non-hematologic characteristics and baseline chronic opioid dose. American Journal of Hematology. https://doi.org/10.1002/ ajh.25168.

15. Williams, H., Silva, S., Cline, D., Freiermuth, C., \& Tanabe, P. (2018). Social and behavioral factors in sickle cell disease: Employment predicts decreased health care utilization. Journal of Health
Care for the Poor and Underserved. https://doi.org/10.1353/ hpu.2018.0060.

16. Badawy, S. M., Thompson, A. A., Lai, J.-S., Penedo, F. J., Rychlik, K., \& Liem, R. I. (2017). Health-related quality of life and adherence to hydroxyurea in adolescents and young adults with sickle cell disease. Pediatric Blood \& Cancer. https://doi.org/10.1002/pbc.26369.

17. Badawy, S. M., Thompson, A. A., Holl, J. L., Penedo, F. J., \& Liem, R. I. (2018). Healthcare utilization and hydroxyurea adherence in youth with sickle cell disease. Pediatric Hematology and Oncology. https://doi.org/10.1080/08880018.2018.1505988.

18. Shah, N., Bhor, M., Xie, L., Paulose, J., \& Yuce, H. (2019). Sickle cell disease complications: Prevalence and resource utilization. PLOS ONE. https://doi.org/10.1371/journal.pone.0214355.

19. Treadwell, M., Telfair, J., Gibson, R. W., Johnson, S., \& Osunkwo, I. (2011). Transition from pediatric to adult care in sickle cell disease: Establishing evidence-based practice and directions for research. American Journal of Hematology. https://doi.org/10.1002/ajh.21880

20. U.S. Food and Drug Administration. (2014). The voice of the patient: Sickle cell disease: A series of reports from the U.S. Food and Drug Administration's Patient-Focused Drug Development Initiative. Retrieved 15 February, 2019, from https://www.fda. gov/downloads/ForIndustry/UserFees/PrescriptionDrugUserFee/ UCM418430.pdf.

21. Smith, W. R., Penberthy, L. T., Bovbjerg, V. E., McClish, D. K., Roberts, J. D., Dahman, B., et al. (2008). Daily assessment of pain in adults with sickle cell disease. Annals of Internal Medicine. https ://doi.org/10.7326/0003-4819-148-2-200801150-00004.

22. Maxwell, K., Streetly, A., \& Bevan, D. (1999). Experiences of hospital care and treatment seeking for pain from sickle cell disease: Qualitative study. BMJ (Clinical Research Ed). https://doi.org/10.1136/ bmj.318.7198.1585.

23. Keller, S., Yang, M., Evensen, C., \& Cowans, T. (2017). ASCQ-Me user's manual. Washington, DC: American Institutes for Research.

24. Keller, S. D., Yang, M., Treadwell, M. J., Werner, E. M., \& Hassell, K. L. (2014). Patient reports of health outcome for adults living with sickle cell disease: Development and testing of the ASCQ-Me item banks. Health and Quality of Life Outcomes. https://doi.org/10.1186/ s12955-014-0125-0.

25. Keller, S., Yang, M., Treadwell, M. J., \& Hassell, K. L. (2017). Sensitivity of alternative measures of functioning and wellbeing for adults with sickle cell disease: Comparison of PROMIS $®$ to ASCQMe ${ }^{\mathrm{SM}}$. Health and Quality of Life Outcomes. https://doi.org/10.1186/ s12955-017-0661-5.

26. Cooper, O., McBain, H., Tangayi, S., Telfer, P., Tsitsikas, D., Yardumian, A., et al. (2019). Psychometric analysis of the adult sickle cell quality of life measurement information system (ACSQ-Me) in a UK population. Health and Quality of Life Outcomes. https://doi. org/10.1186/s12955-019-1136-7.

27. Reilly, M. C., Zbrozek, A. S., \& Dukes, E. (1993). The validity and reproducibility of a work productivity and activity impairment measure. PharmacoEconomics, 4(5), 353-365.

28. Revicki, D., Hays, R. D., Cella, D., \& Sloan, J. (2008). Recommended methods for determining responsiveness and minimally important differences for patient-reported outcomes. Journal of Clinical Epidemiology. https://doi.org/10.1016/j.jclinepi.2007.03.012.

29. Kronborg, C., Handberg, G., \& Axelsen, F. (2009). Health care costs, work productivity and activity impairment in non-malignant chronic pain patients. The European Journal of Health Economics. https://doi.org/10.1007/s10198-008-0096-3.

30. Frederix, G. W. J., Quadri, N., Hövels, A. M., van de Wetering, F. T., Tamminga, H., Schellens, J. H. M., et al. (2013). Utility and work productivity data for economic evaluation of breast cancer therapies in the Netherlands and Sweden. Clinical Therapeutics. https://doi. org/10.1016/j.clinthera.2013.03.009. 
31. Jenerette, C. M., \& Brewer, C. (2010). Health-related stigma in young adults with sickle cell disease. Journal of the National Medical Association, 102(11), 1050-1055.

32. Adegbola, M. (2011). Spirituality, self-efficacy, and quality of life among adults with sickle cell disease. Southern Online Journal of Nursing Research, 11(1).

33. Treadwell, M. J., Hassell, K., Levine, R., \& Keller, S. (2014). Adult sickle cell quality-of-life measurement information system (ASCQ$\mathrm{Me}$ ): Conceptual model based on review of the literature and formative research. The Clinical Journal of Pain. https://doi.org/10.1097/ AJP.0000000000000054.
34. Hassell, K. L. (2010). Population estimates of sickle cell disease in the US. American Journal of Preventive Medicine. https://doi. org/10.1016/j.amepre.2009.12.022.

Publisher's Note Springer Nature remains neutral with regard to jurisdictional claims in published maps and institutional affiliations. 